\title{
WISDOM: Web Intra-page Informative Structure Mining based on Document Object Model
}

\author{
Hung-Yu Kao, Jan-Ming Ho*, and Ming-Syan $\mathrm{Chen}^{+}$ \\ Department of Computer Science \\ and Information Engineering \\ National Cheng Kung University \\ Tainan, Taiwan, ROC \\ E-Mail: hykao@mail.ncku.edu.tw \\ *Institute of Information Science \\ Academia Sinica \\ Taipei, Taiwan, ROC \\ E-Mail: hoho@iis.sinica.edu.tw \\ ${ }^{+}$Electrical Engineering \\ Department \\ National Taiwan University \\ Taipei, Taiwan, ROC \\ E-Mail: mschen@cc.ee.ntu.edu.tw
}

\begin{abstract}
To increase the commercial value and accessibility of pages, most content sites tend to publish their pages with intra-site redundant information, such as navigation panels, advertisements and copyright announcements. Such redundant information increases the index size of general search engines and causes page topics to drift. In this paper, we study the problem of mining intra-page informative structure in news Web sites in order to find and eliminate redundant information. Note that intra-page informative structure is a sub-set of the original Web page and is composed of a set of fine-grained and informative blocks. The intra-page informative structures of pages in a news Web site contain only anchors linking to news pages or bodies of news articles. We propose an intra-page informative structure mining system called WISDOM (Web Intra-page Informative Structure Mining based on the Document Object Model) which applies Information Theory to DOM tree knowledge in order to build the structure. WISDOM splits a DOM tree into many small sub-trees and applies a top-down informative block searching algorithm to select a set of candidate informative blocks. The structure is built by expanding the set using proposed merging methods. Experiments on several real news Web sites show high precision and recall rates which validates WISDOM's practical applicability.
\end{abstract}

\section{KEYWORDS}

Intra-page informative structure, DOM, entropy, information extraction 


\section{INTRODUCTION}

Many Web pages are generated online for Web site maintenance, flexibility, and scalability purposes. They are usually generated by putting page content stored in back-end databases into predefined templates. The experimental results in [4] show that, on average, $43 \%$ of Web pages contain templates which indicates how pervasive template usage has become. Most commercial Web sites, such as search engines, portal sites, e-commerce stores, and news, apply a systematic technique to generate Web pages and to adapt various requests from numerous Web users. These sites are referred to as systematic Web sites [16]. The evolution of automatic Web page generation and the sharp increase of systematic Web sites have contributed to the explosive growth of Web page numbers. There exists much redundant and irrelevant information in these Web pages [1][23], such as navigation panels, advertisements, catalogs of services and announcements of copyright and privacy policies which are distributed over almost all pages of a systematic Web site. Such information is still crawled and indexed by search engines and information agents, thus significantly increasing corresponding storage and computing overhead.

We define specific regions of a page that users are interested in as informative blocks (or referred to as $I B)$. Information within $I B s$ manifests the main topic of the page and indicates related information. The set of these blocks and corresponding connecting structures form the informative structure (or referred to as $I S$ ) of the page. Figure 1 shows the $I S$ of an example news page and its corresponding parts of content. A Web page can be represented by a tree structure, i.e., Document Object Model (DOM) [27] and each content block in a page is a sub-tree of the original DOM tree. The $I S$ can be defined as a reduced tree united by sub-trees of $I B \mathrm{~s}$. The tree relation of united sub-trees is also kept in $I S$. The $I S$ for example in Figure 1 is built by uniting sub-trees of two news table of content blocks and is a tree reduced from the original DOM tree. As proposed in [16], which deals with the IS in a site, called interpage informative structure. The structure is composed of informative pages within a Web site and 
interconnecting links. In this paper, we work with ISS of individual pages, called intra-page informative structure (for simplicity, we use the same denotation $I S$ in the last parts of the paper). Each page has its own $I S$ and the structure is composed of $I B$ s within the page.
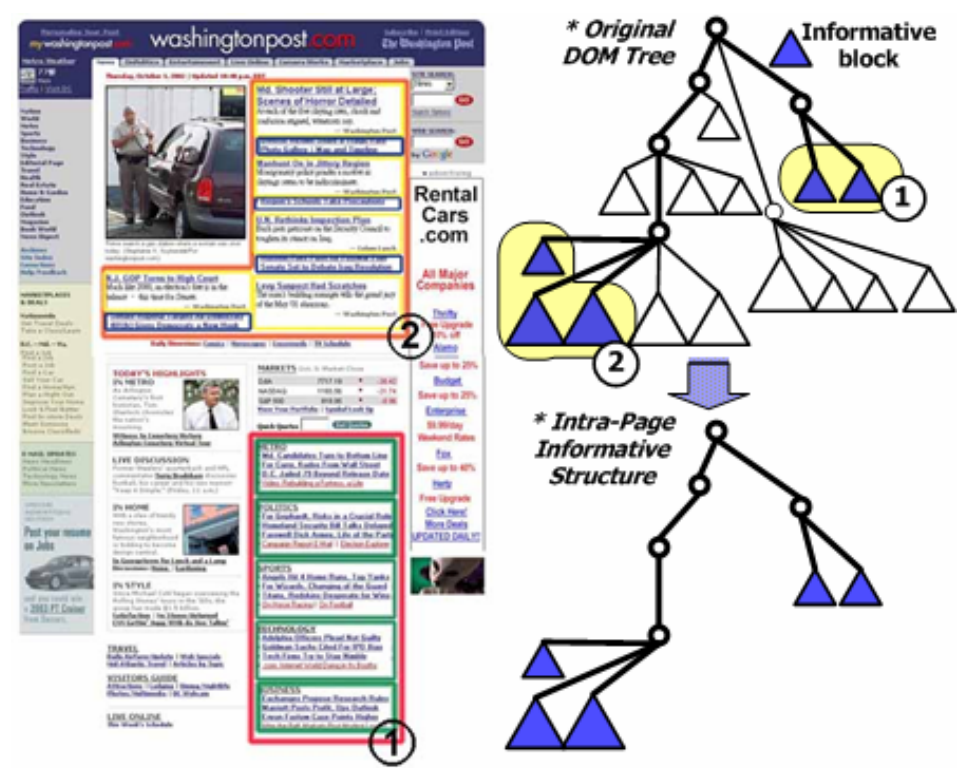

Figure 1: The original document structure and its $I S$ of a news page from WashingtonPost.

Web informative content mining is an important task for search engines and Web agents [11]. Internet crawlers can use the $I S$ to focus on crawling informative paths. Search engines can reduce the size of indices and make them more precise by removing the redundant and irrelevant page blocks. Inter-media information agents that search for specific information among Web sites with different presentation styles, page layouts and site mapping can also benefit from the information pre-processed by the structure.

News search engines like Google News, Altavista News and $\mathrm{NSE}^{1}$, are typical examples of inter-media information agents. They crawl diverse news articles from thousands of news Web sites and extract and index article blocks. The $I S$ of a page consists of sets of table of contents (abbreviated as TOC) blocks 
and news article blocks. The structure helps agents to automatize crawling and indexing. The $I S$ of article pages usually consists of fine-grained and joined $I B S$ and most of them contain only one HTML tag in its sub-tree, i.e., leaf nodes in the DOM tree. These blocks contain news article metadata, such as title, date, reporter and place, which are very useful in categorizing pages for news information agents and metadata extraction. Agents can automatically extract article metadata by using this structure.

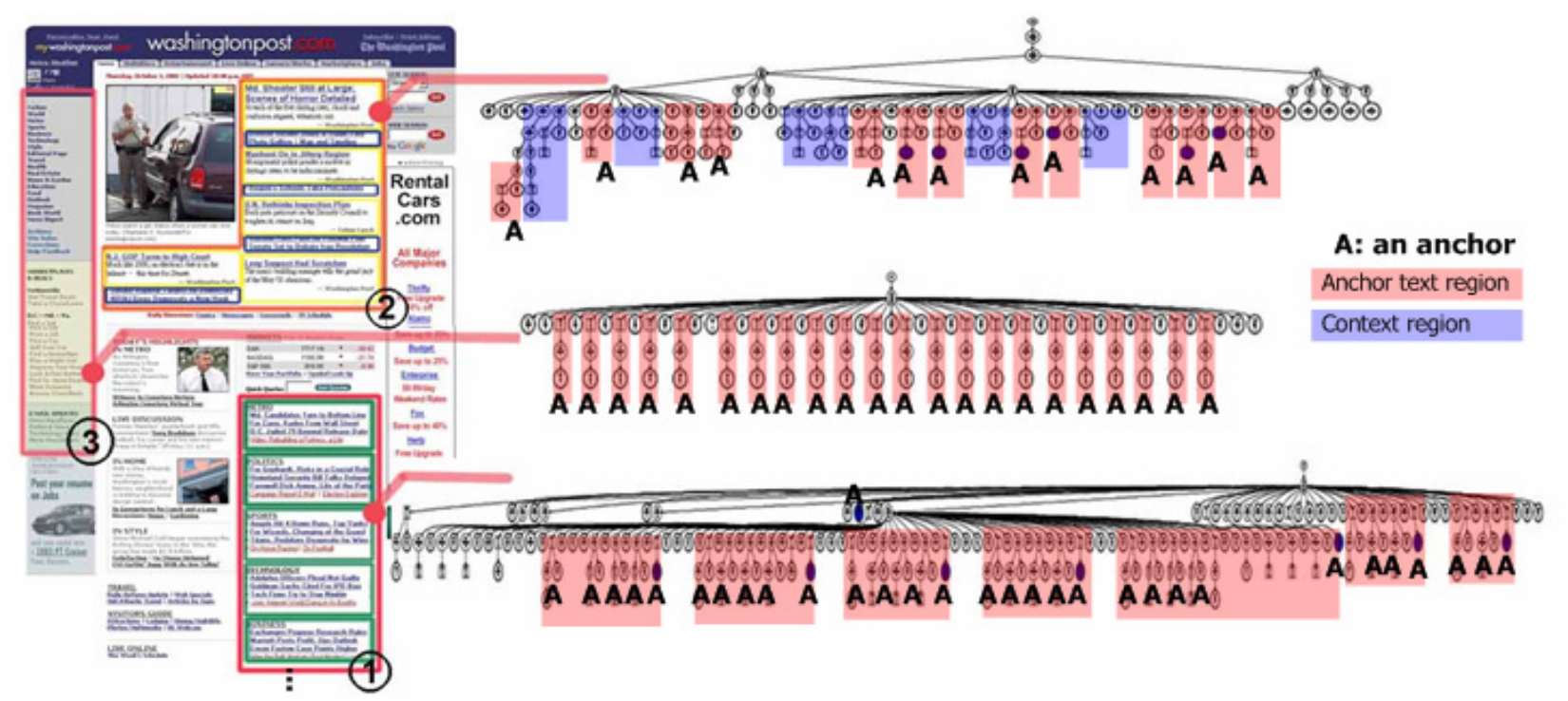

Figure 2: A sample news page from WashingtonPost and the tree structures of informative blocks.

According to the definitions of hubs and authorities in [18], a good hub is a page linking to a good authority page that is relevant to some specific query. Analogously, we define a good information hub as a block linking to a good information authority block which will provide useful information. The IS of a page can then be considered as the set of blocks of good information hubs and good information

\footnotetext{
${ }^{1}$ Google News: http://news.google.com, Altavista News: http://www.altavista.com/news, NSE: http://nse.iis.sinica.edu.tw.
} 
authorities within that page. Note that Web pages in news Web sites usually contain the obvious and clear ISs, i.e., TOC and article blocks, in our observation. Figure 2 shows the root page of the news Web site WashingtonPost (http://www.washingtonpost.com) and blocks 1 and 2 which provide anchors linking to hot news and selected news are the information hubs. We consider these two blocks as $I B s$ as they are the crawling points for news information agents to collect daily news. Block 3 is merely a menu block which is appended ubiquitously to most pages in the WahsingtonPost Web site, and is thus considered as redundant.

In an HTML document, tags are inserted for purposes of the page layout, content presentation and for providing interactive functions, e.g., form filling and document linking. After being rendered by the browser, tags are invisible to users and are represented by means of visual appearances and functions. The layout and style of presentations provide hints to users for accessing and understanding information easily. The corresponding tagging structure therefore contains information about representation and semantics of Web pages. For example, a group of tight sibling anchor nodes with the short anchor-text, e.g., the tagging tree of block 3 in Figure 2, is different from a group of sibling nodes with the long anchor-text interleaved with context nodes, e.g., the tagging tree of block 2 in Figure 2. In Figure 2, block 1 containing several tightly coupled anchor groups also provides different functionality and representation from block 2 and block 3. In news Web sites, a TOC block containing categorized news is usually similar in structure to that of block 1 . Block 2 is also an informative TOC containing the abstracts of news and anchors linking to the news articles. Such useful evidences are more prominent in pages of the systematic Web sites in which ISS are usually generated automatically and dynamically by an iterative program from predefined templates.

In this paper, we extract and use knowledge from the tagging tree structure of a Web page and apply the Information Theory to mine the $I S$. Considering the structure information and context in these nodes 
together, we are able to understand and extract the meaning of information contained in Web pages more clearly and precisely. Specifically, we propose in the paper an IS mining system called WISDOM, standing for Web Intra-page Informative Structure Mining based on the Document Object Model to automatically extract and recognize the $I S$ of each page in a Web site.

The main mining flow in WISDOM first uses the Information Theory to evaluate the information amount contained in each DOM tree node, and then constructs the $I S$ by applying the specific searching, filtering and merging methods. The searching step finds $I B$ candidates and its principle is based on the observation that the root node of an IB uniformly spreads its information around its children nodes in most cases. In view of this, WISDOM first splits the original DOM tree into several small and nonoverlapped sub-trees as shown in Figure 3 and selects some of them as the candidate sub-trees, in accordance with the assigned threshold of the structure information. The threshold is applied for the judgment on the uniformity of information distribution. Some uninformative sub-trees are removed in the step. Our system then applies a top-down $I B$ searching algorithm to find the top-k most informative blocks and the corresponding filtering criteria to select a set of candidate ISs called the skeleton set. The skeleton set can be considered the core sub-trees of the $I S$ shown in the color shaded regions in Figure 3 . The $I S$ is built by expanding the skeleton set using the proposed merging methods. The merging method works in a bottom-up manner to link the qualified sibling nodes in the skeleton set and other informative nodes.

The remainder of this paper is organized as follows. In Section 2, we describe related work. WISDOM is described in Section 3. In Section 4, we evaluate the performance of WISDOM by testing it on several real news Web sites, university and commercial Web sites. The Section 5 gives our conclusion. 


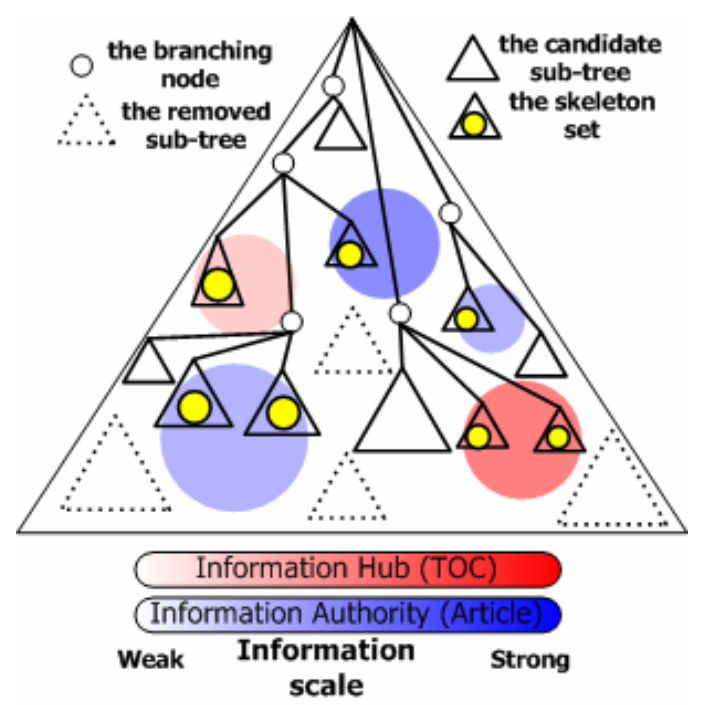

Figure 3: The tree splitting and selection in WISDOM.

\section{RELATED WORK}

Many works have been proposed that aim to extract the information of a page. Works on wrappers [9][19][22] provide learning mechanisms to mine the extraction rules of documents. The WebKB project in [6] automatically creates a computer understandable knowledge base from the textual content and hyperlink connectivity of Web pages. The work describes an inductive logic programming algorithm for learning wrappers and develops a trainable information extraction system. Works in [1][15][20] provide auxiliary systems to aid in the information extraction from semi-structured documents. The clipping method proposed in [14] is based on a supervised learning to provide a practical tool to cut the news articles. However, they need either a pre-marked training set or a considerable amount of human involvement to perform information extraction. When we consider the whole World Wide Web as our problem domain, building a useful training set to represent the diversity of Web content and structure is very hard.

In a systematic Web site, IBs are usually generated by a loop program; the entities in blocks are therefore similar to one another in view of their tag patterns and information they carry. In Figure 2, it 
can be seen that the tag patterns of micro blocks (the shaded regions) in IBS 1 and 2 look very similar to one another. Therefore, frequent substructure mining is a candidate solution for automatic extraction of $I B$ s. The topic of mining frequent substructure on the DOM trees of semistructure pages has recently been studied in [2][10][24] where the frequent sub-tree was extracted by respective pattern mining and noise node concealment methods, such as the wildcard mechanism in [10] and node-skip and edge-skip pruning in [2]. Works also use the tree pattern mining to extract metadata information in Web pages [13][28]. However, semantic information in mined blocks with the same tree structure may be different from one to another. We need other information measurement methods to filter out redundant information blocks from those blocks with similar tree structure. Moreover, some $I B$ s like article blocks, are laid out with the unique structures and are indeed difficult to extract by the frequent structure mining. Some techniques proposed in [12][29] use the semantics and relationships of tags to extract the record boundaries of Web pages. Several heuristic rules of tag characteristics, such as the highest count-tags (HT), identifiable "separator" tag (IT) and repeating tag pattern (RP), are proposed in [12] and are applied to extract record boundaries on several ".com" Web sites. Research in [29] also categorized tags into several groups according to their tagging functionalities and discovered the major schemas between them to translate HTML documents to XML documents in a semantic view.

Research in [7] extends the definition of a hub by dividing a hub page into several fine-grained hub blocks with different hub values. This is accomplished by calculating and integrating the hub values of each anchor node in the DOM tree of a page. Entropy analysis proposed in [21] discriminates the informative authorities of pages by dividing a page into several authority blocks with different authority values weighted by the information of each block.

There are also works on mining informative structure [16][21], which are different from our work in that they mainly deal with mining blocks delimited by $<$ TABLE $>$ tags. In contrast, we mine fine-grained 
blocks using the DOM tree. It is worth mentioning that in the problem of mining the fine-grained $I B$ s in a page, a straightforward approach would be to divide the page into several unit blocks that contain only one tag and then to merge neighboring blocks that contain information together. This naive method, however, does not work well for real-world Web pages in our opinion, because (1) when an $I B$ is divided into small blocks with the one-tag granularity, the information contained is also divided into many small pieces which are difficult to discriminate from noises and redundant information, and (2) there is no obvious method to merge such small blocks to form meaningful and integrated $I B s$. We therefore propose a top-down mining instead of bottom-up algorithm to extract fine-grained $I B$ s.

\section{WISDOM: A DOM BASED MINING SYSTEM}

WISDOM automatically extracts and recognizes ISS of each page in a Web site according to the knowledge in the tree structures of pages. As shown in Figure 4, WISDOM consists of three phases: (1) information extraction from DOM trees, (2) $k$-maximum informative block mining, and (3) block expansion and condensation. In the first phase, we extract useful features from the information of the original DOM tree. These features can be classified into two types of information: node information and structure information. In the second phase, we aggregate the node information to build the Information Coverage Tree (ICT). According to the $I C T$, we devise a greedy algorithm, i.e., $k$-maximum informative block mining algorithm ( $k$-MIB), to extract sub-trees that contain richer information. The extracted subtrees are either better information hubs or better information authorities, depending on the criteria employed in the greedy algorithm. They form the skeleton set of the $I S$ of a page. We then expand the skeleton set by assembling neighboring sub-trees that contain similar features corresponding to the original skeleton sub-trees. After condensing the expanded set by removing dummy nodes, the assembled forest (or tree), in essence the $I S$ of a page, is constructed. 


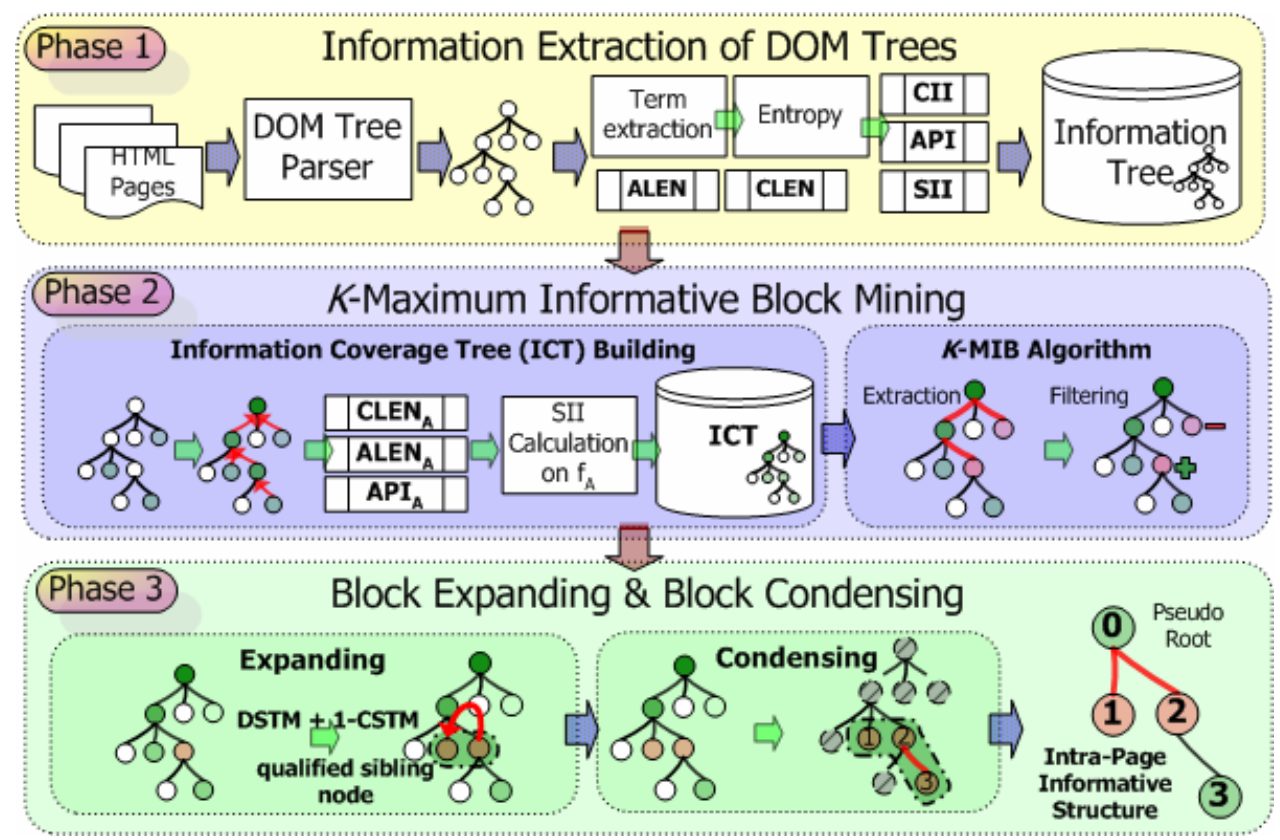

Figure 4: WISDOM system flow.

\subsection{Phase 1: Information Extraction from DOM Trees}

In the beginning, we crawl pages of a Web site in a specific crawling depth. When a page is crawled, we first extract the tree structure of a page based on DOM. Note that some HTML pages are not wellconformed, e.g., missing the ending $</ a>$ tag for the $<\mathrm{a}>$ tag. We use HTMLTidy ${ }^{2}$ to fix syntax mistakes in source documents. In tree $T$, each node represents a tag in the page and contains the tag name information, attributes in the tag statement and its innerText, i.e., the context delimited by the tag. From the definition of DOM, the context of the innerText of node $N$ includes all contexts of nodes in the subtree rooted by node $N$. We use $T(N)$ to denote the sub-tree rooted by node $N$. The innerText of the root node in each page is the context of a page when all tags are removed. The text of a Web page can be classified into two types: (1) anchor texts and (2) contexts which are texts delimited by all other tags

\footnotetext{
${ }^{2}$ HTMLTidy is an HTML fixing tool developed by Dave Raggett from the W3C team, http://www.w3.org/People/Raggett/tidy/.
} 
except $<\mathrm{A}>$ tags. We use $A L E N$ to represent the length of the anchor text of a node and CLEN to represent the length of the contexts. A list of symbols used in this paper is given in Table 1.

Table 1: The list of symbols used.

\begin{tabular}{|l|l||l|l|}
\hline Abbr. & Description & Abbr. & Description \\
\hline \hline ALEN & length of anchor text & ALEN $_{\mathbf{A}}$ & aggregated ALEN \\
\hline CLEN & length of contexts & CLEN $_{\mathbf{A}}$ & aggregated CLEN \\
\hline API & anchor precision index & API $_{\mathbf{A}}$ & aggregated API \\
\hline $\mathbf{F}$ & $\begin{array}{l}\text { the set of tuple values, } \\
\text { (ALEN, CLEN, API) }\end{array}$ & $\mathbf{F}_{\mathbf{A}}$ & $\begin{array}{l}\text { the set of aggregated tuple values, } \\
(\text { ALEN }\end{array}$, CLEN API $\left._{\mathrm{A}}\right)$
\end{tabular}

We then parse the innerText of the root node to extract meaningful terms. A term corresponds to a meaningful keyword or phrase. Applying stemming algorithms and removing stop words based on a stop-list, English keywords (terms) can be extracted in a systematic manner [26]. Extracting terms in oriental languages is more difficult because of the lack of separators in these languages. In our system, we use an algorithm to extract keywords from Chinese sentences based on a Chinese term base. This base was generated by our search engine ${ }^{3}$ by collecting hot queries and excluding stop words. After extracting terms in all crawled pages, we calculate the entropy value of each term according to its term frequency. From Shannon's information entropy [25], the entropy of term $\operatorname{term}_{i}$ can be formulated as:

$$
\operatorname{EN}\left(\text { term }_{i}\right)=-\sum_{j=1}^{n} w_{i j} \log _{n} w_{i j} \text {, where } w_{i j}>0 \text { and } n=|D|, D \text { is the set of pages, }
$$

where $w_{i j}$ is the value of normalized term frequency in the page set. In the experiments on real Web sites containing a huge amount of pages, it is not practical to re-calculate entropy values directly when a new 
page is crawled. In WISDOM, we uses an incremental entropy calculation process in the real Web site analysis. In the incremental calculation process, the new entropy value $E_{k+1}\left(f_{j}\right)$ is calculated only by the previous entropy value $E_{k}\left(f_{j}\right)$, total term frequency $T F_{j, k}$ and the new term frequency $t f_{(k+1) j}$ of the new included page for term $f_{j}$. The incremental calculation can be described as $E_{k+1}\left(f_{j}\right)=\left\{\begin{array}{l}\frac{E_{k}\left(f_{j}\right)}{\log _{k}(k+1)}, \text { when } t f_{(k+1) j}=0 \\ \Phi\left(E_{k}\left(f_{j}\right), \quad T F_{j, k}, \quad t f_{(k+1) j}\right), \text { otherwise }\end{array}\right.$. The proof of the correctness of the process is given in Appendix A.

We define the weight of a term $T_{j}$ as $\mathrm{W}\left(T_{j}\right)=1-E N\left(T_{j}\right)$ to represent the importance of the term. The reason behind of applying entropy calculation is that terms distributed in more pages in a Web site usually carry less information to users. In contrast, those appearing in fewer pages carry more information of interest. The weight of a term is similar to its inverse document frequency, IDF [3], which is defined as $\log _{n} \frac{n}{d f_{j}}$, where $d f_{j}$ is the document frequency of $T_{j}$. IDF is usually applied to represent the discriminability of a term in a set of documents. According to the definition, we can conclude following relationships between $\mathrm{W}\left(T_{j}\right)$ and $I D F_{j}$ : (1) If $T_{j}$ is uniformly distributed among some pages, $\mathrm{W}\left(T_{j}\right)=$ $I D F_{j}$, and (2) If $T_{j}$ is not uniformly distributed among the same pages in (1), then $\mathrm{W}\left(T_{j}\right)>I D F_{j}$ and the more skewed the distribution of $T_{j}$ is, the larger $\mathrm{W}\left(T_{j}\right)$ is. The two relationships are proved in [17] and we include the detail of proofs in Appendix A. Benefiting from these two relationships, the weight of a term attained from the entropy value is more representative for the importance of a term than from IDF. We use the example illustrated in Figure 5 to explain these relationships. In this figure, Term $_{\mathrm{A}}$ is uniformly distributed among Page 1 to Page 3 and $\operatorname{Term}_{B}$ has the same term frequency and the

\footnotetext{
${ }^{3}$ The searching service is a project sponsored by Yam, a commercial search engine in Taiwan (http://www.yam.com/).
} 
document count with $\mathrm{Term}_{\mathrm{A}}$, but most $\mathrm{Term}_{\mathrm{B}} \mathrm{S}$ are located at Page 3. These two relationships are conformed by the following calculations.

$$
\left\{\begin{array}{l}
W\left(\operatorname{Term}_{A}\right)=1-\operatorname{EN}\left(\text { Term }_{A}\right)=1+3 * \frac{2}{6} \log _{4} \frac{2}{6}=0.207519=\operatorname{IDF}\left(\text { Term }_{A}\right)=\log _{4} \frac{4}{3}=0.207519 \cdots(1) \\
W\left(\operatorname{Term}_{B}\right)=1-E N\left(\text { Term }_{B}\right)=1+2 * \frac{1}{6} \log _{4} \frac{1}{6}+\frac{4}{6} \log _{4} \frac{4}{6}=0.374185>I D F\left(\text { Term }_{B}\right)=\log _{4} \frac{4}{3}=0.207519 \cdots(2)
\end{array}\right.
$$

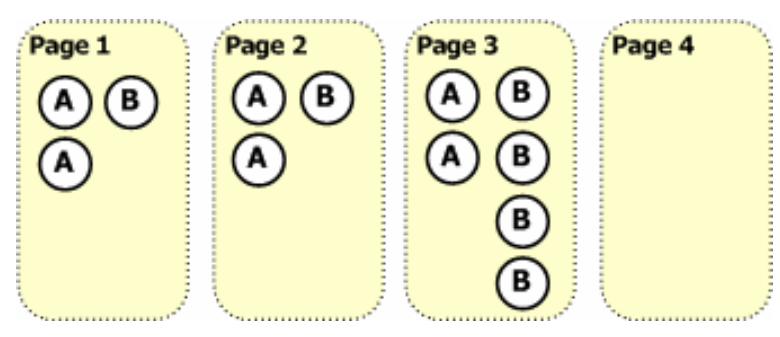

Figure 5: An example of different term distributions

According to the extracted information, we calculate three extended features to gain more implicit information from the tree, namely (1) the content information index $(\boldsymbol{C I I})$ which indicates the amount of information contained in the block, (2) the anchor precision index $(\boldsymbol{A P I})$ which represents the similarity between the anchor-text and the linked document, and (3) the structure information index (SII) which indicates the distribution of children's feature values of one node in the DOM tree. Each node in DOM tree $T$ contains the tuple values of the feature set $\boldsymbol{F}=\{A L E N, C L E N, A P I\}$. In the following sections, we will describe their respective calculations.

\subsubsection{Content information indices (CII)}

When entropy values of terms are calculated, we average the weight values of terms in an innerText of node $N$ to get the content information index of $N$, i.e.,

$$
C I I(N)=\frac{\sum_{j=1}^{k} W\left(\text { term }_{j}\right)}{k}, \text { where } \forall_{j=1 \sim k} \text { term }_{j} \text { in innerText of } N \text {. }
$$


The $C I I$ value of node $N$ represents the amount of information carried in a sub-tree rooted by $N$. The works in [16][21] have shown that the entropy value corresponds to the recognition of the context parts of article pages. Consider the $C I I$ distribution of an article page in Figure 6(a). Note that the DOM tree is built by a depth-first traversal. The node ID of each node in the tree is generated according to the traversal order. Nodes with close node IDs are adjacent to each other in the physical layout of the page. The shaded region is an $I B$ of the page, which is identified manually. Observed that (1) nodes with higher $C I I$ values in an $I B$ are more than others and (2) nodes in an $I B$ form a clear cluster in the $C I I$ distribution graph.

\subsubsection{Anchor precision indices (API)}

When browsing the Web, people use anchors to get information they want according to the semantics of anchors. The semantics of an anchor can be represented by the anchor text, text surrounding the anchor, the image or other dynamic representations generated by scripts. The semantics of an anchor is expected to be relative to the page it links. Such relevance is, however, weak in some cases. We therefore define the value of the anchor precision index to indicate the correlation of the anchor and its linking page. We use the anchor text and the bounded text surrounding the anchor to evaluate the value of $A P I$. The correlation index $A P I$ is defined as:

$$
A P I(N)=\sum_{j=1}^{m} \frac{1}{E N\left(\text { term }_{j}\right)},
$$

where $\operatorname{term}_{j}$ is the term concurrently appearing in both the anchor text of $N$ and the linked page and $m$ is the number of matched terms.

The calculation of API stems from the similarity analysis between documents using the vector space model. We extend the model by using the inverse values of entropy to set the weights of terms. If the information amount in those matched terms is larger, we get a larger API value that indicates that the 
anchor carries more precise information. The usage of the inverse of entropy values in the API formulation is to emphasize and amplify the effect of matched terms. Moreover, the value of $A P I$ is not normalized by the matched count, because we want to show that the longer informative anchor text leads to more information. Note that $E N\left(\right.$ term $\left._{i}\right)$ is always larger than 0 because $\operatorname{term}_{i}$ appears in at least two documents.

Consider the API distribution of a TOC page in Figure 6(b). It shows that the number of nodes with larger API values in the shaded region, i.e., regions of marked TOC blocks, are more than others on average. Anchors in the menu block have small $A P I$ values, because the anchor texts of these anchors are short and the entropies of terms they contain are almost one.

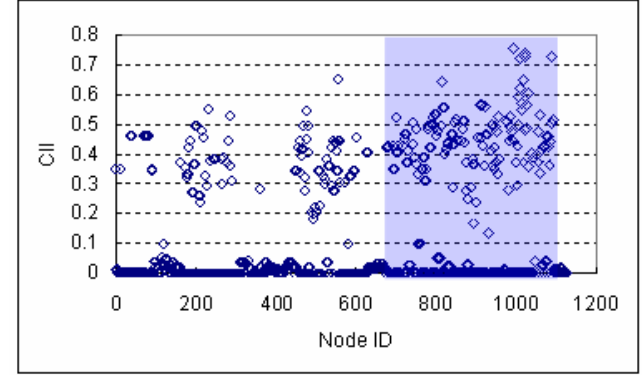

(a)

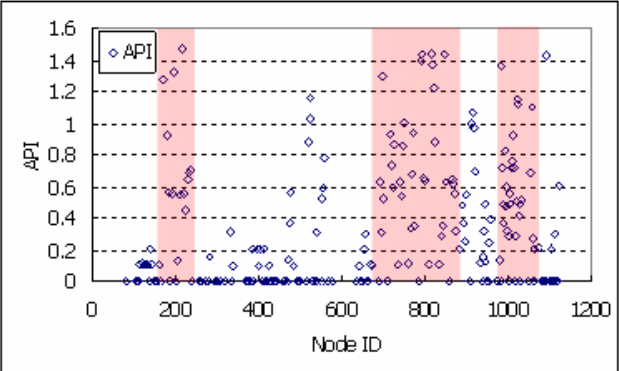

(b)

Figure 6: The distributions of CII and API for two sample Web pages (shaded regions are IBs identified from the answer sets).

\subsubsection{Structure information indices (SII)}

The index SII of a node is calculated according to the distribution of the feature values of the node's children. However, some HTML tags either correspond to information that is not extractable or provide no useful information. Such tags, such as the comment tag $<$ ! $>$, the new line tag $<$ br $>$ and the script program tag $<$ script $>$, are called dummy tags and are removed from the following calculation of SII. We define the notion $f_{i}(N)$ as the value of feature $f_{i}$ of node $N$, and children $(N)$ as the set of all non-dummy children of the node $N$. For a simple tree structure of node $N$ with children $n_{0}, n_{1}, \ldots, n_{m-1}$, we define the SII value of node $N$ for feature $f_{i}$ as: 


$$
\operatorname{SII}\left(N, f_{i}\right)=-\sum_{j=0}^{m-1} w_{i j} \log _{m} w_{i j}, \text { where } w_{i j}=\frac{f_{i}\left(n_{j}\right)}{\sum_{k=0 \sim m-1} f_{i}\left(n_{k}\right)}, \forall n_{k} \in \operatorname{children}(N) .
$$

Note that $f_{i}(N)$ is larger or equal to the sum of $f_{i}\left(n_{0}\right), f_{i}\left(n_{1}\right), \ldots, f_{i}\left(n_{m-1}\right)$. We apply entropy calculation here to represent the distribution of children's feature values of any node with more than one child. The value of SII indicates the degree that the feature values of the node are dispersed among its children. When the value of $\operatorname{SII}\left(N, f_{i}\right)$ is higher, the values of all children's $f_{i}$ tend to be equal.

In a systematic Web site, most context and anchors of TOC blocks are generated automatically. The styles, appearances and information carried of entities in such a block are always similar from one to another. This phenomenon makes the SII values of these features become larger ones for the root nodes of such blocks.

\subsection{Phase 2: The $k$-Maximum Informative Block Mining}

In this phase, we first build the information coverage tree for features extracted during the phase one to obtain corresponding aggregated feature values. The proposed $k$-MIB algorithm is then applied to extract and filter out the candidate $I B$ s. In Section 3.2.1, we describe the construction of $I C T$ and the aggregated features. Extracting and filtering processes of the proposed algorithm are described in Section 3.2.2.

\subsubsection{Information Coverage Tree Building}

We define a tree with bottom-up aggregated features as an information coverage tree (abbreviated as $I C T)$. In an $I C T$, any feature in the aggregated feature set $\boldsymbol{F}_{\boldsymbol{A}}$ is obtained from the corresponding features in set $\boldsymbol{F}$. Each node in an ICT contains all feature information of nodes in the sub-tree rooted by this node. The feature aggregation is a bottom-up process from the leaf nodes to the root node. The process of level $k$ of the tree is shown below: 


$$
f_{A i}(N)=f_{i}(N)+\sum f_{A i}\left(n_{j}\right), \forall n_{j} \in \operatorname{children}(N) \text { and level }(N)=k
$$

We aggregate features from the lowest level of the tree to the level one. The complexity of the process is $\mathrm{O}(|\mathrm{N}|)$. Figure 7 shows an example aggregation process where the node marked by $*$ is labeled with $6=3+(3+0)$ and the one marked by $* *$ is labeled with $8=1+(1+6)$.

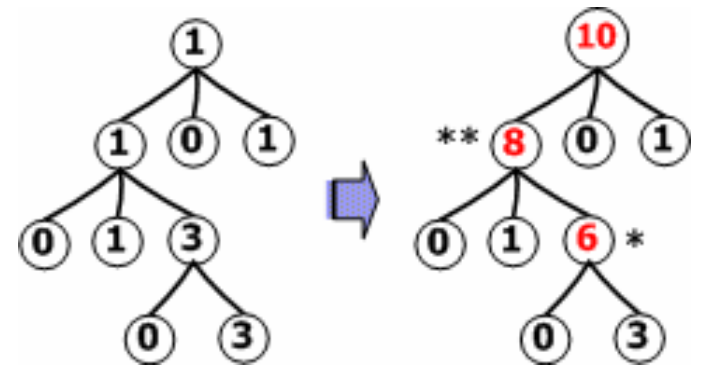

Figure 7: An example of feature aggregation.

The aggregated features in ICT for each node $N$ are subject to the constraint where $f_{A i}\left(n_{j}\right)$ is the aggregated value of feature $f_{i}$ of node $n_{j}$ :

$$
f_{A i}(N) \geq \sum_{j=0}^{m-1} f_{A i}\left(n_{j}\right), \forall n_{j} \in \operatorname{children}(N)
$$

The length of innerText of each node is a typical aggregated feature because the innerText of a parent node contains all the innerText of its child nodes. We use $T L E N_{A}$ to represent the length of innerText. In WISDOM, we also aggregate node information $A L E N$ and $A P I$ to get the corresponding aggregated features, denoted by $A L E N_{A}$ and $A P I_{A}$. Note that $\operatorname{TLEN}_{A}(N)$ is composed of the length of contexts in $T(N)$, i.e., $C L E N_{A}(N)$, and the length of anchor texts in $T(N)$, i.e., $A L E N_{A}(N)$. The value of $T L E N_{A}$ is thus equal to $C L E N_{A}+A L E N_{A}$. We then apply SII calculation on these three aggregated features to get corresponding structure information of aggregated features for each node. 


\subsubsection{Block extracting and block filtering}

The proposed maximum informative block mining algorithm $M I B\left(\boldsymbol{k}, \boldsymbol{f}_{\boldsymbol{A}}, \boldsymbol{S T}\right)$ is a greedy and top-down tree traversal process. For input value $k$, the algorithm outputs at most $k I B$ s, i.e., TOC blocks or article blocks. The aim of the algorithm is to find the top- $k$ nodes with maximal aggregated feature $f_{A}$ values under the given SII constraint, i.e., SII Threshold $(\boldsymbol{S T})$. When the value of $S T$ is larger, the structure constraint is tighter and the children of each extracted node in the resulting candidate set will have more similar values of aggregated features in accordance with the definition of SII. The searching path of the algorithm is shown for example in Figure 8. The original tree is extracted from a real TOC page by eliminating those sub-trees removed by $M I B$.

When extracting the top- $k$ candidate nodes, we apply type constraints to eliminate pseudo-informative nodes. Type constraints (TC) are dependent on the type of blocks described as:

$$
\left\{\begin{array}{l}
\text { if type }=" \text { Article", } C I I(N) \geq 1-T C_{\text {article }} \\
\text { if type }=" T O C ", \frac{A P I_{A}(N)}{\# \text { anchors in } T(N)} \geq T C_{T O C}
\end{array}, \forall N \in\right. \text { CandidateSet. }
$$

Type constraints are motivated from heuristic observations that (1) article blocks contain informative context and hence their entropy values must be bounded and (2) TOC blocks contain highly semantic relevant anchors linking to information authorities and the average $A P I$ value should be more than others in blocks with redundant and irrelevant anchors. These heuristic constraints are useful in removing pseudo-informative blocks.

Due to the tree traversal characteristic of the MIB algorithm, each node in the filtered candidate set is not an ancestor of any other nodes. The sub-trees rooted by these nodes are therefore isolated and nonoverlapped. The set of these selected sub-trees is called the skeleton of the $I S$ of a page and the root nodes of these sub-trees skeleton nodes. 

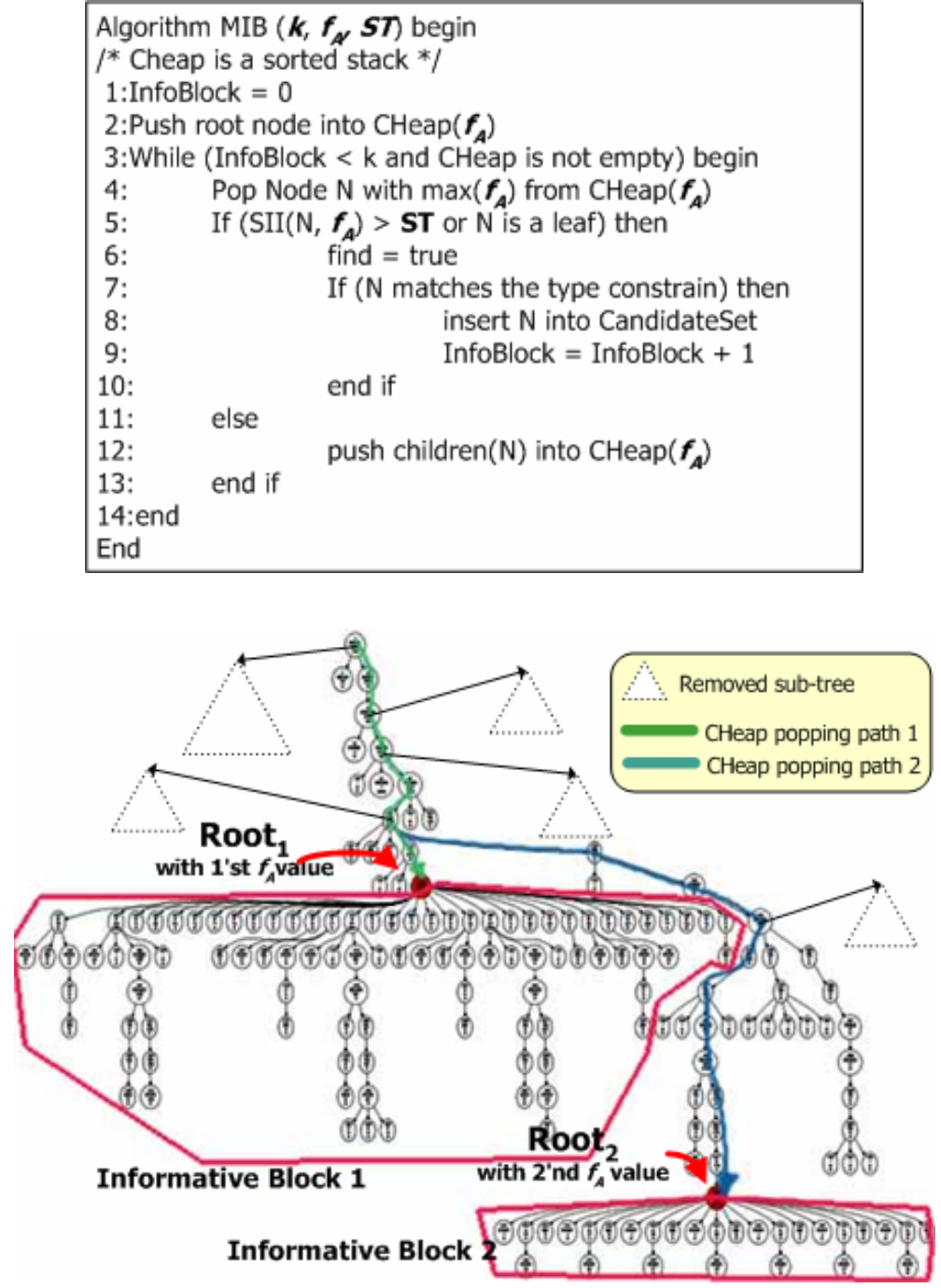

Figure 8: An example of $k$-maximum informative block mining on the tree of a TOC page, $k=2$.

\subsection{Phase 3: Block Expanding and Condensing}

When investigating on the skeleton set, we find the selected skeleton nodes are often the sub-trees of the $I B$ s. The observation is more obvious when the structure threshold (ST) is larger and tighter. This is because the selected sub-tree is smaller when ST becomes larger in the k-MIB process. According to the skeleton structure, we therefore apply two sibling tree merging methods, i.e., direct sibling tree merging $(D S T M)$ and collateral sibling tree merging (CSTM), to expand the skeleton set. The DSTM method merges the sub-tree rooted by qualified sibling nodes of each skeleton node $S$ as shown in Figure 9 . 
Note that node $S$ ' may be one of skeleton nodes. Any qualified sibling node $S$ ' needs to match the type constraints and $f_{A}\left(S^{\prime}\right)$ must also be smaller than $f_{A}(S)$. We do not need to merge sibling nodes with the larger $f_{A}$ values, because they are checked in the previous searching paths of the $k$-MIB algorithm and have been either selected into the skeleton set or removed from the IS. After DSTM, we then select the non-singleton ancestors, i.e., $P_{1}, P_{2}, \ldots, P_{n}$, of $S$ for the process of CSTM. The $i$-th non-singleton ancestor $P_{i}$ is the $i$-th ancestor of $S$ which has more than one non-dummy sibling node. The dummy node is defined as a node whose value of $f_{A}$ is zero, e.g., the node with $C L E N_{A}=0$ for the article block and the node with $A L E N_{A}=0$ for the TOC block. The method of $k$-CSTM is equal to applying DSTM on $P_{k}$. In WISDOM, we apply DSTM and 1-CSTM to proceed the default block expanding. For example, in Figure 9, we merge sub-trees rooted by three $S^{\prime}$ into the skeleton set in the DSTM process. In the 1 CSTM process, we first traverse the tree from the node $S$ up to the root node to find the first nonsingleton ancestor $P_{1}$ and we then apply $D S T M$ on $P_{1}$ to merge its qualified sibling nodes, i.e., two $P_{1}$, nodes.

The intention of DSTM is to merge small $I B$ s surrounding the skeleton blocks together. The non-uniform distribution between $f_{A}$ values of the skeleton node and corresponding sibling nodes leads to the node separation in the $k-M I B$ phase. In our experiments, DSTM can merge the metadata blocks, i.e., the article title, date, reporter, etc., into the main body of the article news. They are all $I B \mathrm{~s}$, but the distribution of their context length is skewed.

The block condensing process removes the sub-trees rooted by nodes that cannot match the type constraints from the expanding trees as these dummy sub-trees are mainly tags for the page layout. This process is used to remove the uninformative sub-blocks from the merged trees obtained from the previous processes. 


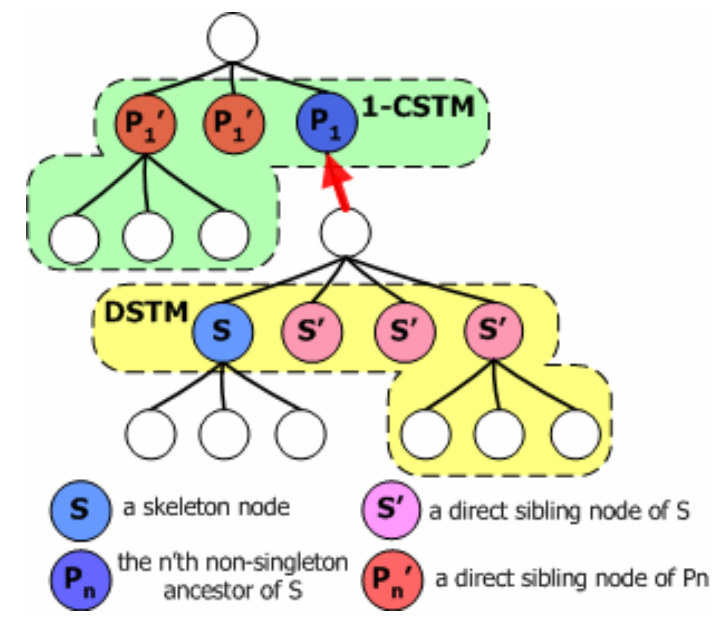

Figure 9: Two sibling tree merging methods.

Table 2: Datasets and their informative structure distributions.

\begin{tabular}{|c|c|c|c|c|c|c|c|c|c|}
\hline \multirow[t]{2}{*}{ Site Abbr. } & \multirow[t]{2}{*}{ URL } & \multirow{2}{*}{$\begin{array}{l}\text { Total } \\
\text { pages }\end{array}$} & \multirow{2}{*}{$\begin{array}{c}\text { TOC } \\
\text { pages }\end{array}$} & \multirow{2}{*}{$\begin{array}{c}\text { Marked } \\
\text { TOC } \\
\text { pages }\end{array}$} & \multirow{2}{*}{$\begin{array}{c}\text { Marked } \\
\text { article } \\
\text { pages }\end{array}$} & \multirow{2}{*}{$\begin{array}{c}\text { Marked } \\
\text { Toc } \\
\text { blocks }\end{array}$} & \multirow{2}{*}{$\begin{array}{c}\text { Marked } \\
\text { article } \\
\text { blocks }\end{array}$} & \multicolumn{2}{|c|}{ Answer Coverage } \\
\hline & & & & & & & & TOC (S-NC) & Article (IC) \\
\hline $\mathrm{CDN}$ & www.cdn.com.tw & 261 & 25 & $22^{*}$ & $60^{\#}$ & 38 & 63 & $46.30 \%$ & $98.40 \%$ \\
\hline CTIMES & news.chinatimes.com & 3747 & 79 & 69 & 66 & 313 & 68 & $32.10 \%$ & $82.50 \%$ \\
\hline CNA & www.cna.com.tw & 1400 & 33 & 29 & 50 & 106 & 50 & $21.90 \%$ & $80.10 \%$ \\
\hline CNET & taiwan.cnet.com & 4331 & 78 & 38 & 37 & 84 & 86 & $17.50 \%$ & $63.60 \%$ \\
\hline CTS & www.cts.com.tw & 1316 & 31 & 19 & 53 & 21 & 80 & $54.80 \%$ & $52.10 \%$ \\
\hline TVBS & www.tvbs.com.tw & 740 & 13 & 12 & 50 & 25 & 50 & $73.70 \%$ & $56.90 \%$ \\
\hline TTV & www.ttv.com.tw & 861 & 22 & 18 & 42 & 20 & 75 & $20.10 \%$ & $54.50 \%$ \\
\hline UDN & udnnews.com & 4676 & 252 & 243 & 52 & 674 & 106 & $28.00 \%$ & $67.80 \%$ \\
\hline CORN & www.cs.cornell.edu & 1346 & $\mathrm{~N} / \mathrm{A}^{\mathrm{S}}$ & 14 & 14 & 24 & 18 & $45.42 \%$ & $80.80 \%$ \\
\hline UTEX & www.cs.utexas.edu & 2935 & N/A & 11 & 10 & 11 & 10 & $45.02 \%$ & $84.90 \%$ \\
\hline WASH & www.cs.washington.edu & 1526 & N/A & 16 & 10 & 23 & 10 & $79.04 \%$ & $69.98 \%$ \\
\hline WISC & www.cs.wisc.edu & 2973 & N/A & 10 & 15 & 11 & 15 & $41.73 \%$ & $77.08 \%$ \\
\hline ABOUT & compnetworking.about.com & 498 & N/A & 10 & 10 & 11 & 48 & $18.65 \%$ & $43.54 \%$ \\
\hline ECNET & reviews.cnet.com & 500 & N/A & 10 & 10 & 10 & 10 & $38.89 \%$ & $57.72 \%$ \\
\hline ESPN & sports.espn.go.com & 494 & N/A & 8 & 10 & 16 & 12 & $28.32 \%$ & $58.39 \%$ \\
\hline MONET & www.mo.net & 261 & N/A & 9 & 10 & 9 & 13 & $35.78 \%$ & $54.91 \%$ \\
\hline XML & www.xml.com & 807 & $\mathrm{~N} / \mathrm{A}$ & 10 & 10 & 10 & 26 & $43.46 \%$ & $80.89 \%$ \\
\hline
\end{tabular}

\section{EXPERIMENTS AND RESULTS}

In this section, we describe several experiments conducted on some real news Web sites in order to evaluate the performance of WISDOM. Datasets used and employed evaluation criteria are described in Section 4.1. We evaluate the performance of selection and filtering in the $k$-MIB algorithm in Section 0. 
Performance of block expanding and condensing is assessed in Section 4.3. Finally, Section 4.4 provides the overall performance evaluation of WISDOM.

\subsection{Datasets}

We conduct our experiments on the datasets ${ }^{4}$ used in [16]. In addition to these news Web sites, for evaluating WISDOM on other domains, a good example is the dataset used in the WebKB project [8] and the dataset used in the page segmentation research [9]. These datasets contain several university sites and commercial Web sites as described in Table 2. To assess WISDOM, we add two new answer sets, i.e., TOC blocks and article blocks. These blocks are extracted manually by news domain experts ${ }^{5}$ according to their experience in issuing real-world newspapers. We select most TOC pages and some candidate pages among all article pages with different tagging structures to mark. Unmarked TOC pages are pages which cannot be correctly parsed, or those containing many out-site anchors linking to uncrawled pages, e.g., TOC pages in CNET and TTV. The latter case will cause the accuracy of API calculation to decrease suddenly and blur the evaluation results.

As shown in Table 2, the percentages of information coverage of the $I S$ over the original page vary among datasets. Values are dependent on the styles and page layouts of news sites. The more redundant information added, the less information the $I S$ carries.

To attain a quantitative evaluation, we employ two different evaluating methods to measure the values of precision and recall of article and TOC blocks. The TOC evaluation method is called significant node coverage (SNC). In SNC, we count the matched anchor nodes in sub-trees rooted by nodes in the answer set and our output. For evaluating article blocks, we calculate the ratio of the matched context contained

\footnotetext{
${ }^{4}$ Pages of Web sites in datasets were crawled on 2001/12/27, 2002/4/11 and 2004/3/29. The datasets can be retrieved at our research site http://kp06.iis.sinica.edu.tw/isd/index.html.

${ }^{5}$ The news domain experts are researchers at the Department of Journalism, National Chengchi University, Taiwan.
} 
in each sub-tree by length to indicate the performance. The method is called information coverage (IC). The selection is made because only the context and anchors in the $I S$ need to be indexed and extracted for crawling. In our experiments, we use the rates of precision $(\mathrm{P})$ and recall $(\mathrm{R})$ to indicate the similarity of these two sets. We also use F-measure [3] which combines recall and precision in a single efficiency measure. The value is the harmonic mean of precision and recall, and is formulated as $\frac{2 *\left(R^{*} P\right)}{R+P}$. With the example in Figure 10, we show the evaluation results of four methods in Table 3 . The answer sets are two sets of root nodes, i.e., the TOC answer set $\mathrm{A}_{T}=\left\{a_{T 1}, a_{T 2}, \cdots, a_{T n}\right\}$ and the article answer set $\mathrm{A}_{A}=\left\{a_{A 1}, a_{A 2}, \cdots, a_{A n}\right\}$. The extracted results are the set of TOC blocks $W_{T}=\left\{w_{T 1}, w_{T 2}, \cdots, w_{T n}\right\}$ and the set of article blocks $W_{A}=\left\{w_{A 1}, w_{A 2}, \cdots, w_{A n}\right\}$.

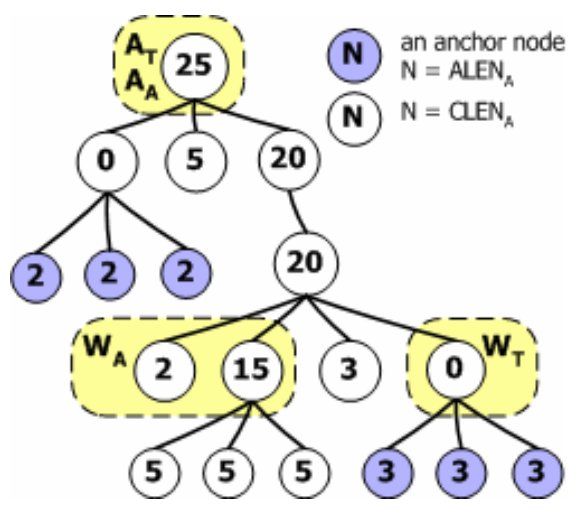

Figure 10: A simple tree with an answer node and two results marked.

Table 3: The evaluating calculation of the example in Figure 10.

\begin{tabular}{|c|c|c|c|c|c|c|c|c|c|c|c|}
\hline \multicolumn{7}{|c|}{$\operatorname{Method}\left(\mathrm{A}_{\mathrm{T}}, \mathrm{W}_{\mathrm{T}}\right)$} & \multicolumn{5}{|c|}{$\left(\mathrm{A}_{\mathrm{A}}, \mathrm{W}_{\mathrm{A}}\right)$} \\
\hline & $\mathrm{AW}$ & $\mathrm{AO}$ & \begin{tabular}{l|l} 
WO & $\mathrm{P}$ \\
\end{tabular} & & $\mathrm{R}$ & $\mathrm{F}$ & $\mathrm{AW}$ & $\mathrm{AO}$ & WO $\mathrm{P}$ & $\mathrm{R}$ & $\mathrm{F}$ \\
\hline SNC & 3 & 3 & $\begin{array}{ll}0 \\
\end{array}$ & 1 & 0.50 & 0.67 & 5 & 5 & 0 & \begin{tabular}{l|l|}
1 & 0.50
\end{tabular} & 0.67 \\
\hline IC & 9 & 6 & 0 & 1 & 0.60 & 0.75 & 17 & 8 & 0 & \begin{tabular}{l|l|}
1 & 0.68
\end{tabular} & 0.81 \\
\hline $\begin{array}{l}* \mathrm{AW}= \\
* \mathrm{AO}=\mathrm{t} \\
* \mathrm{WO}= \\
* \mathrm{P}=1\end{array}$ & $\begin{array}{l}\text { nun } \\
\text { nul } \\
\text { //(A }\end{array}$ & $\begin{array}{l}\text { nber } \\
\text { mber } \\
W+\mathrm{V}\end{array}$ & $\begin{array}{l}\text { of ansv } \\
\text { of ans } \\
\text { of ansv } \\
\text { vO), } \mathrm{R}=\end{array}$ & & $\begin{array}{l}\text { of the } \\
n \mathrm{Ab} \\
\text { in } \mathrm{W} \\
\mathrm{V} /(\mathrm{AV}\end{array}$ & $\begin{array}{l}\text { inters } \\
\text { but not } \\
\text { but no } \\
\mathrm{W}+\mathrm{AO}\end{array}$ & $\begin{array}{l}\text { section } \\
t \text { in W } \\
\text { ot in A }\end{array}$ & & & & \\
\hline
\end{tabular}


We show the result of the incremental entropy calculation in Figure 11. In the figure, the value of the Yaxis means the ratio of the resulting entropy and the final entropy value calculated from the whole page set. We can find that the ratio difference is smaller than 0.1 when the corresponding document count is larger than 200. Therefore, in the practical usage, WISDOM can achieve a stable performance when the crawled page set is smaller than the whole page set of a Web site.
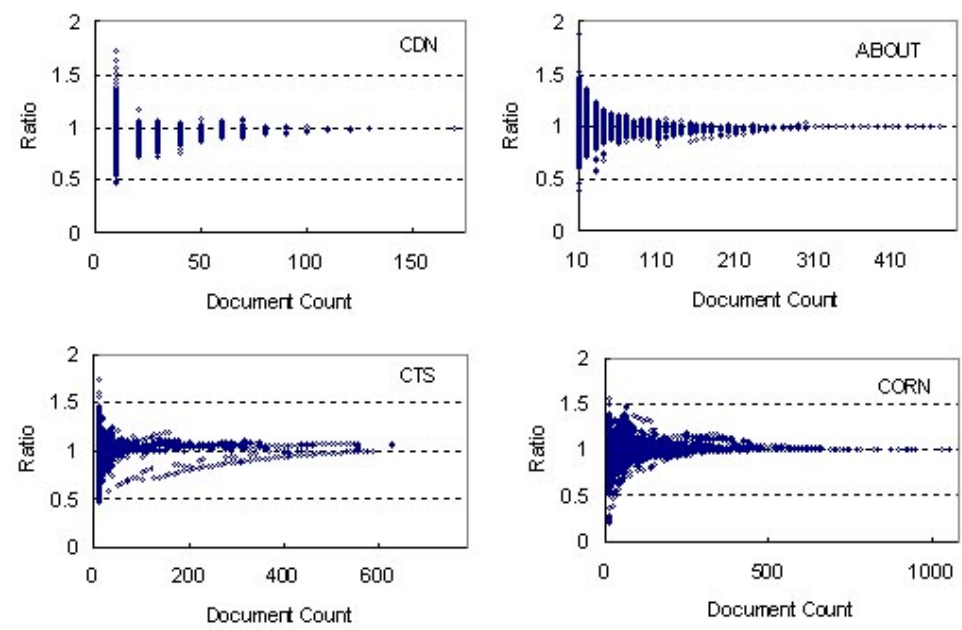

Figure 11: Incremental Entropy distribution for datasets CDN, CTS, ABOUT and CORN.

\subsection{Evaluation of $\boldsymbol{k}$-MIB}

After the ICT of a page is built, we have to determine the searching $\left(f_{A}\right)$ and branching $(S T)$ criteria before applying $k-M I B$ to the $I C T$. These selection criteria of $k-M I B$ will affect the performance of the algorithm. In Figure 12, we first conduct experiments to show the effects of different selection criteria for TOC blocks. We select $A L E N_{A}$ and $A P I_{A}$ for the searching criteria and corresponding $S I I$ values for the branching criteria. The result in Figure 12 shows that using $\operatorname{SII}\left(A P I_{A}\right)$ for the branching criterion outperforms the one using $\operatorname{SII}\left(A L E N_{A}\right)$ when the selection criterion is to use a threshold of being equal to or smaller than 0.8 . This is because $A P I_{A}$ contains more information for discriminating the informative and redundant links than the length of anchor texts does. 


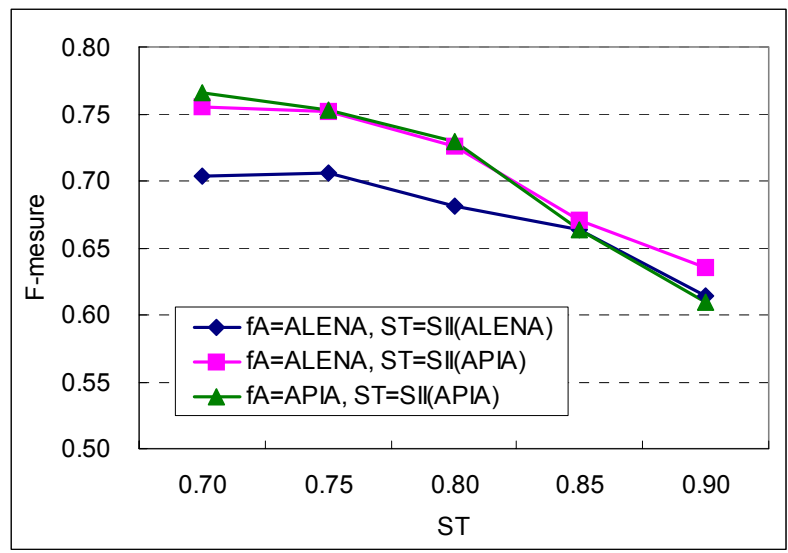

Figure 12: The effect of different criteria of $k$-mib for TOC blocks.

We then apply the $k$-MIB algorithm to the $I C T$ with the parameter pair $\left(k, f_{A}, S T\right)$. We use different $S T$ values to control the number and granularity of the $I B \mathrm{~s}$. When the $S T$ value is larger, more tighter and smaller blocks will be induced as shown in Figure 13. Note that the average size of IBs in CTIMES is about three times as others and is out of the boundary of Figure 13. This is due to the existence of big TOC blocks with entries of all categories of news in CTIMES. The sizes of these blocks are about 800 tags (nodes).

In the second phase of WISDOM, type constraint filtering plays an important role to remove the falsepositive nodes. The selection of $T C_{T O C}$ and $T C_{\text {Article }}$ is made as follows. The distributions of the average $A P I$ values, i.e., the criterion of the TOC type constraint, of top-k $I B \mathrm{~s}$ in UDN are shown in Figure 14 where it can be seen that there are two obvious noise groups of values in this figure, i.e., 1.1 and 2.2, and they are reasonably chosen to be the $T C_{T O C}$. The selection of $T C_{A r t i c l e}$ is not so straightforward as the selection of $T C_{T O C}$. This is because when the size of $I B$ s is divided into smaller ones, the number of extracted terms in each small block decreases, so does the accuracy of corresponding CII. Moreover, the index $C I I$ is not an aggregated value. We choose the uninformative link threshold described in [16], i.e., 0.8 , to be $T C_{\text {Article }}$. Consequently, we use $(1.25,0.8)$ as default values for $\left(T C_{T O C}, T C_{A r t i c l e}\right)$ for all datasets in WISDOM. The choice of $T C_{T O C}$ value, 1.25 , is simply motivated from the $A P I$ formulation. 
We assume that each basic informative link contains one matching term with entropy 0.8 , and its API value is 1.25 by the formulation. The value conforms to our observation on the real data shown in Figure 14.
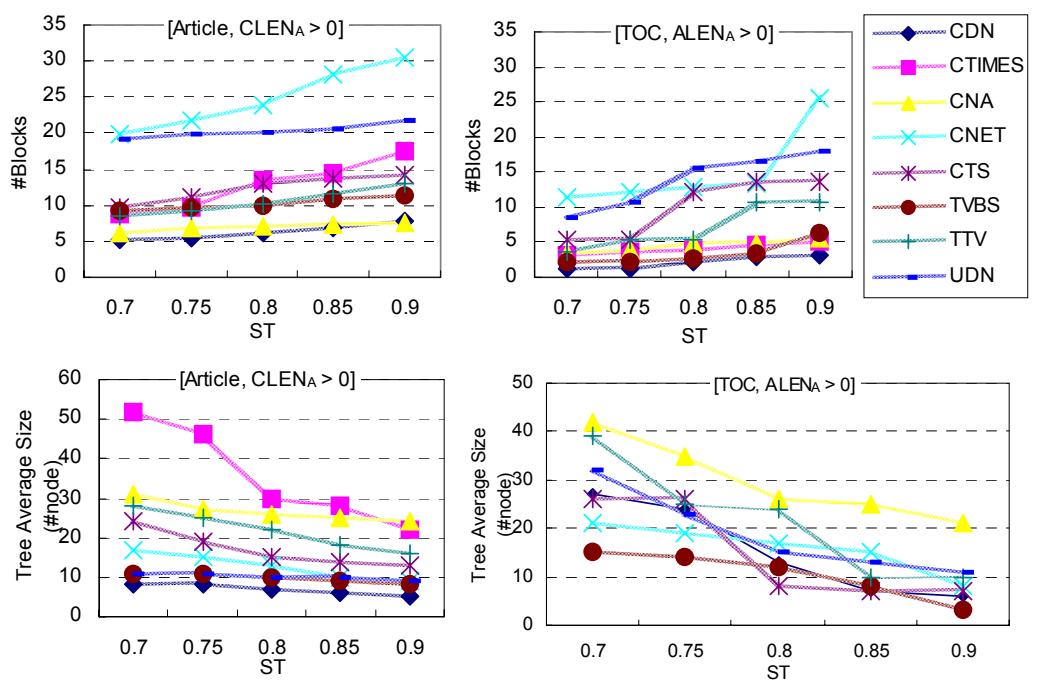

Figure 13: The average number and size of total $I B$ s in a page selected by k-MIB without filtering.

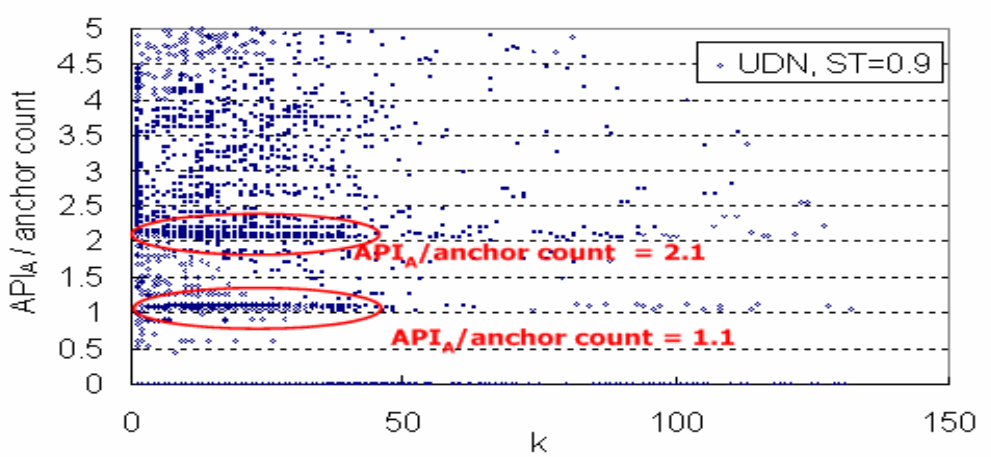

Figure 14: The selection of the type constraint of TOC blocks [UDN, ST=0.9].

We show the average precision and recall values of $k$-MIB under the different selections of $S T$ and $k$ in Figure 15. The results of TOC and article blocks both show the phenomena incurred by $S T$. When the value of ST increases, the sizes of split $I B$ s decrease and the granularities of these blocks become finer. The selection of more fine-grained $I B$ s increases the precision, but reduces the coverage of $I B \mathrm{~s}$, i.e., the recall. The same observation can be made in the same figure when the value of $k$ becomes smaller. 
From the results in Figure 15, WISDOM is good at mining the informative article blocks rather than TOC blocks. First, there exists only one informative article block in most marked article pages. The information of an article page is thus more concentrated than information of a TOC page. This helps WISDOM discriminate informative article blocks easily. Second, noises affect and blur the API value. Using entropy to indicate the amount of information does not work well when few terms are extracted from the anchor text. The local menu effect mentioned in [16] can also decrease the entropy values of anchors in the menu blocks. The noise effects are prominent in CNET and UDN and the discriminability of the $A P I$ value in these Web sites decreases suddenly. In CNET, more than $68 \%$ of all $I B$ s have average $A P I$ values of less than 2 if $\mathrm{k}<=5$. Third, some informative TOC blocks mined by WISDOM are not "news" TOC blocks. These blocks are not selected in the answer set. The effects of applying different TCs are shown in Figure 16. Filtering constraints can be used to remove pseudo-informative blocks.
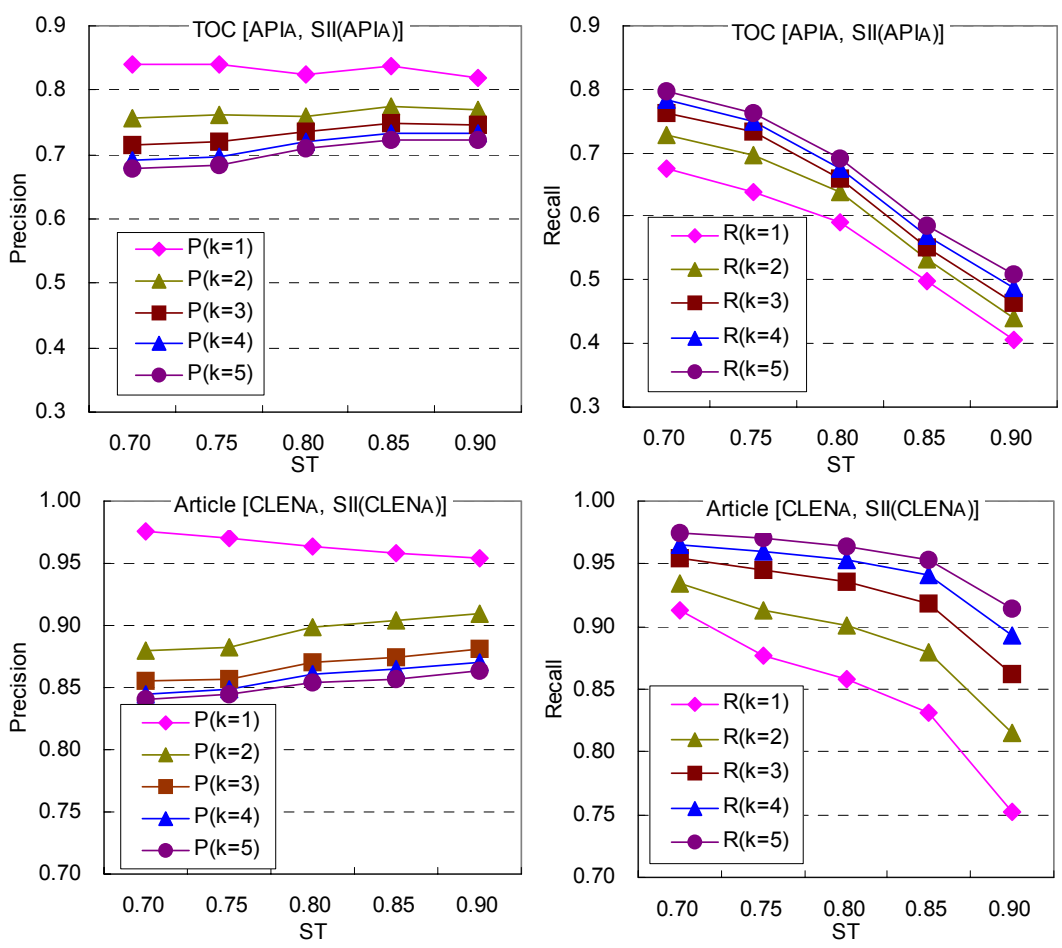

Figure 15: The average values of precision and recall before phase 3 (caption: block type $\left[f_{A}, S I I\right]$ ). 


\subsection{Evaluation of block expanding and condensing}

Figure 17 shows that the average improvement of different merging methods. The performances of experiments with different STs become similar after block expansion. This is because most blocks extracted by a high ST value are real $I B \mathrm{~s}$, though the sizes of these blocks are smaller than blocks extracted by a low ST value. The sizes of these smaller blocks can be expanded to the sizes of larger blocks by merging sibling sub-trees which are also real $I B \mathrm{~s}$. Merging methods do not work well if a skeleton set contains many pseudo-informative blocks, such as TOC blocks in CNET. Expansion of the skeleton set will incur more false-positive results. This is also the reason that the results with $\mathrm{k}=1$ are better than those with $\mathrm{k}=3$.
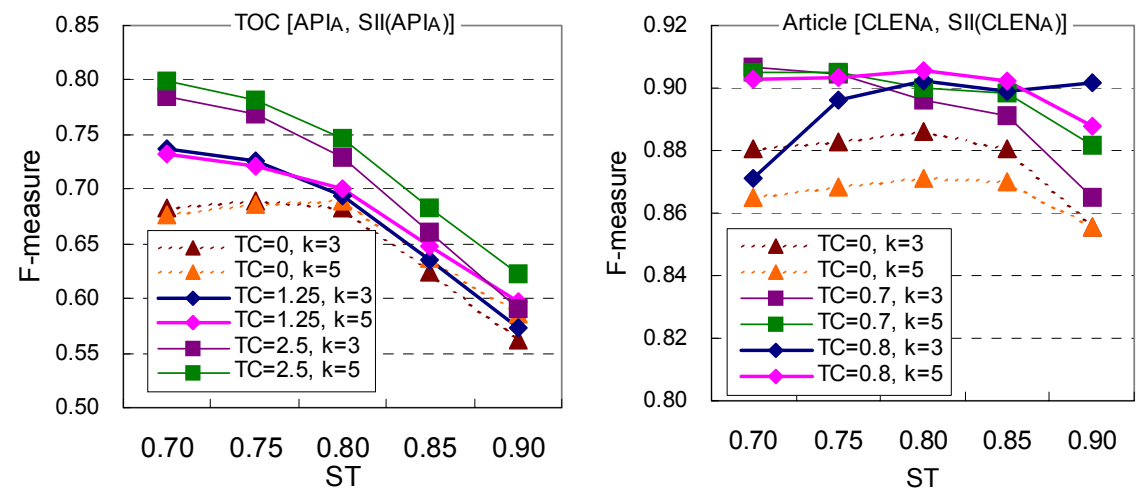

Figure 16: The effects of different type constraints.

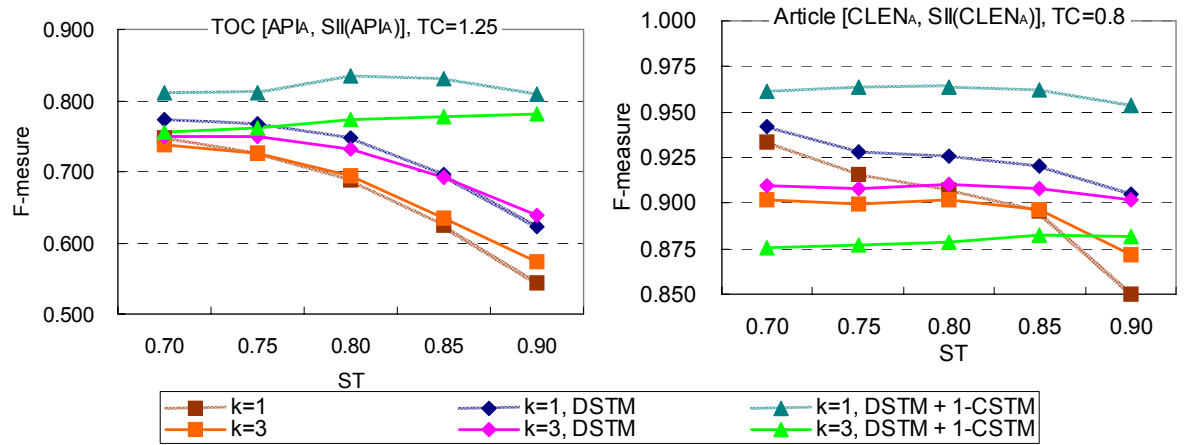

Figure 17: The effects of DSTM and 1-CSTM. 


\subsection{Overall Performance}

In Figure 18, we use the system default setting, i.e., $\mathrm{k}=1, \mathrm{ST}=0.8, \mathrm{TC}=(0.8,1.25)$ and merging methods DSTM and 1-CSTM, to show the overall performance of WISDOM on each dataset. This figure shows that WISDOM is very good at the article blocks mining of all datasets and exhibits excellent performance on TOC blocks mining of CDN, CTIMES, CNA, CTS, and TVBS. The low values of precision and recall on CNET, TTV and UDN are caused by the low accuracy of API values. Another low precision value on UDN is affected by the merging method 1-CSTM. Many pseudo-informative blocks are merged in the 1-CSTM step even though WISDOM has reached the high recall rate after DSTM merging. The high average values of precision and recall also represent the robustness of WISDOM. We also compare WISDOM with two straightforward extracting methods in Figure 19 to show the improvement. The method M1 selects and merges leaf nodes of a DOM tree to a set of subtrees and is similar to the straightforward method described in Section 2. These merged leaf nodes must satisfy the same information constraint of WISDOM. The method M1 can be treated as simplified WISDOM that selects all leaf nodes into the skeleton set in the k-MIB phase. The method M2 works like M1 as well. The difference is that the method M2 uses the length constraint to filter the merged leaf nodes, i.e., the TLEN of a node must be larger than 5. Figure 19 shows that WISDOM with the default setting leads these two methods and gives the prominent performance for the article pages. 


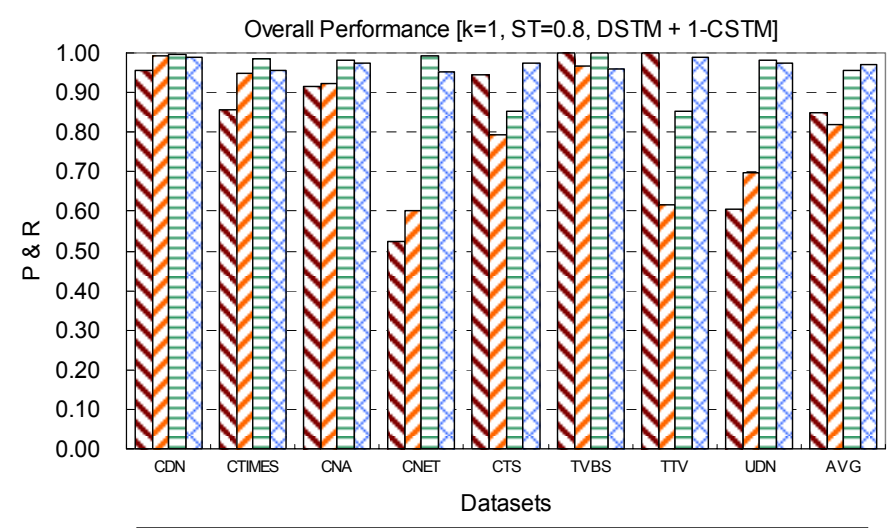

$\square$ TOC Precision $\square$ TOC Recall $\boxminus$ Article Precision $\square$ Article Recall

Figure 18: Overall performance of WISDOM.

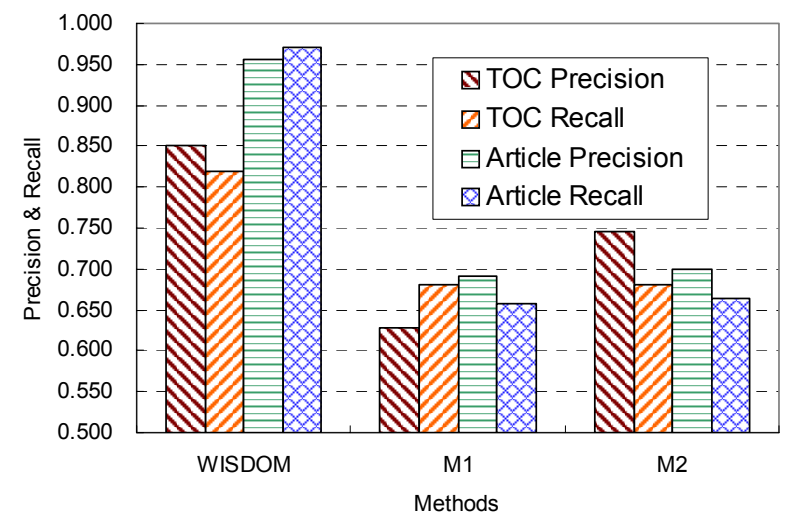

Figure 19: Comparison of WISDOM to two straightforward methods.

The result in Figure 20 shows the overall performance for English Web sites which consist of university and commercial domains by using the default setting same as in news Web sites. The performance for the article block extraction is also good as that in news Web sites. However, the performance of the TOC block extraction is worse than that in news Web sites. We found three reasons to cause the negative effect, which are (1) some informative anchors contains short anchor-text and common terms between anchors and linking pages are few. We cannot extract the anchor information in these cases and the feature APIs of these informative anchors therefore cannot be discriminated from redundant ones, (2) some important terms are considered as stop-words and ignored, e.g., course-id in the university course pages. WISDOM ignores the numerical terms to reduce the noise effect caused by their high weights, 
which are obtained from the entropy calculation. However, course-id is an important clue for users to choice which course they feel interesting. This evidence shows that the stop-words selection must be dependent on the domain characteristics, otherwise some important words will be ignored, (3) many commercial anchors are generated by the script language embedded in the pages. WISDOM cannot extract the anchor texts from these dynamic links.

To remedy these issues, we conduct an experiment to use different features instead of API to extract TOC blocks. The result in Figure 21 shows the improvement when the feature ALEN and corresponding ST threshold are applied on some datasets in which API doest not work well. This can be explained by that the TOC structure characteristics are retained when features ALEN and SII(ALEN) are applied and blurred when API is not correctly calculated or hard to be evaluated due to the lack of matched terms. API values are always zero even though corresponding ALEN values are larger than zero in these situations. We can evaluate the ratio of numbers of zero-API anchors over all meaningful anchors, i.e., their ALEN values are more than some threshold, in a page to be our criterion on the selection of appropriate features. The average ratios of datasets in Figure 21 are obviously higher than others from our experimental observations.

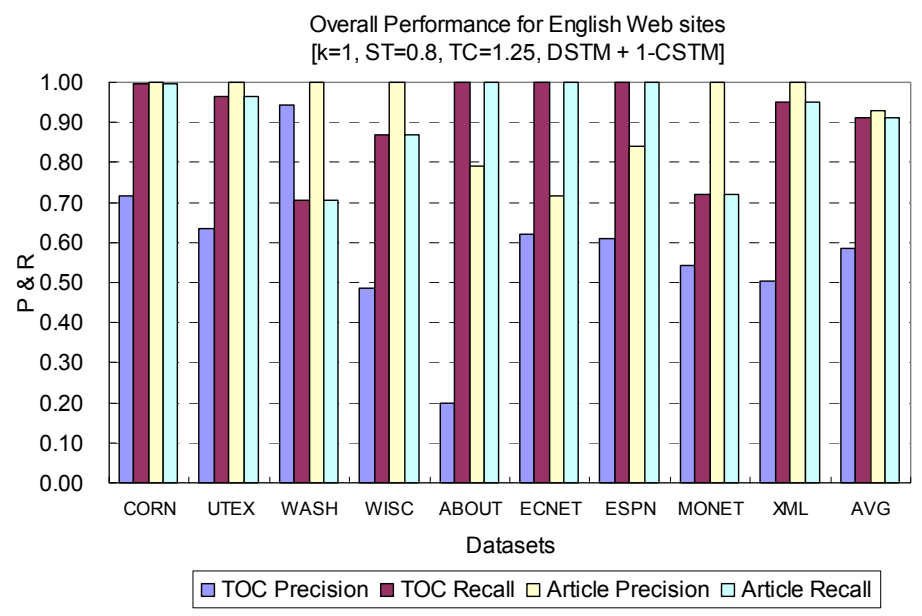

Figure 20: Overall performance of WISDOM on other domain Web sites. 


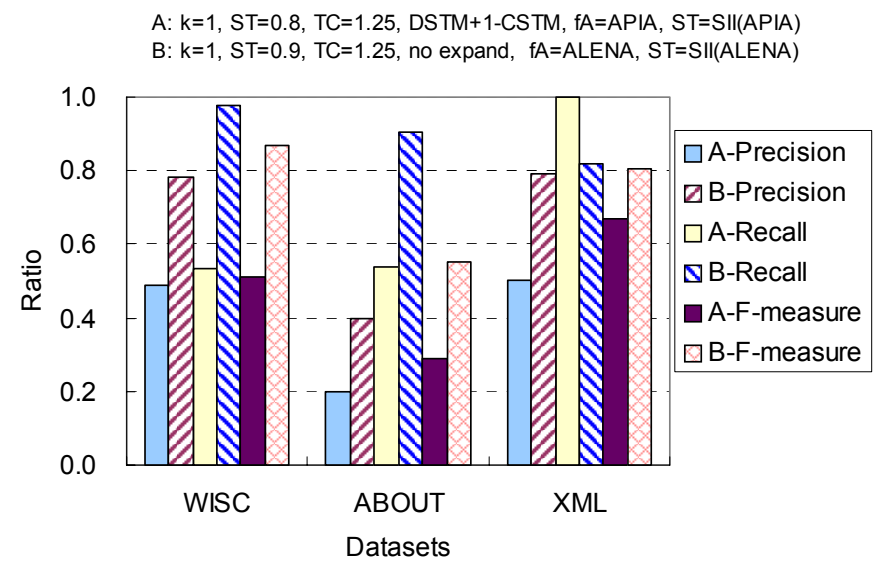

Figure 21: Different feature selection for the TOC extraction.

\section{CONCLUSION}

We propose WISDOM to mine the ISs of a page. Given an entrance URL, WISDOM is able to crawl the site, parse pages into DOM trees and analyze node and structure information in order to build information coverage trees. The system uses the Information Theory to split the DOM tree of a Web page into a set of IBs and uses the proposed searching (k-MIB), filtering and merging (DSTM, 1 -CSTM) methods to mine the $I S$ of a page.

For search engines, inter-media information agents, and crawlers, the $I S$ mined by WISDOM is useful for indexing, extracting and navigating significant information from a Web site. Experiments on several real news Web sites show high precision and recall rates attained by WISDOM which validates its practical applicability on news Web sites. We are integrating WISDOM into our news search engine (NSE) to help system managers speed up their work flow and reduce the labor of maintaining the sitedependent rule based extraction. For Web sites in other domains, even for non-systematic Web sites, we are conducting some augmented feature to remedy the noise effects and improve the applicability. 


\section{REFERENCES}

[1] B. Adelberg. NoDoSE - a tool for semi-automatically extracting structured and semistructured data from text documents. Proc. of the 1998 ACM SIGMOD international Conf. on Management of data (SIGMOD), 1998.

[2] T. Asai, K. Abe, S. Kawasoe, H. Arimura, H. Sakamoto, S. Arikawa. Efficient Substructure Discovery from Large Semi-structured Data. SIAM international Conf. on data mining (SDM), 2002.

[3] R. Baeza-Yates and B. Ribeiro-Neto, Modern Information Retrieval, Addision Wesley, 1999.

[4] Z. Bar-Yossef, S. Rajagopalan, Template Detection via Data Mining and its Applications, Proc. of 11th World Wide Web Conf. (WWW), 2002.

[5] A. Broder, S. Glassman, M. Manasse, G. Zweig. Syntactic Clustering of the Web. Proc. of the 6th World Wide Web Conf. (WWW), 1997.

[6] M. Craven, D. DiPasquo, D. Freitag, A. McCallum, T. Mitchell, K. Nigam, S. Slattery, "Learning to construct knowledge bases from the World Wide Web", Artificial Intelligence, Vol. 118, No. 1-2, pp. $69-113,2000$.

[7] S. Chakrabarti, Integrating the Document Object Model with Hyperlinks for Enhanced Topic Distillation and Information Extraction. Proc. of 10th World Wide Web Conf. (WWW), 2001.

[8] Y. Chen, W.-Y. Ma, H.-J. Zhang, Detecting Web Page Structure for Adaptive Viewing on Small Form Factor Devices, Proc. of 12th World Wide Web Conf. (WWW), 2003.

[9] W. Cohen. Recognizing Structure in Web Pages using Similarity Queries. The National Conf. on Artificial Intelligence (AAAI), 1999.

[10]G. Cong, L. Yi, B. Liu, K. Wang. Discovering Frequent Substructures from Hierarchical Semistructured Data. SIAM International Conf. on Data Mining (SIAM SDM), 2002. 
[11]R. Cooley and J. Srivastava. Web Mining: Information and Pattern Discovery on the World Wide Web. Proc. of the 9th IEEE International Conf. on Tools with Artificial Intelligence (ICTAI), 1997.

[12]D. W. Embley, Y. Jiang and Y. K. Ng. Record-boundary discovery in Web documents. Proc. of the 1999 ACM SIGMOD International Conf. on Management of data (SIGMOD), 1999.

[13]K. Furukawa, T. Uchida, K. Yamada, T. Miyahara, T. Shoudai, Y. Nakamura. Extracting Characteristic Structures among Words in Semistructured Documents. Proc. of the Sixth PacificAsia Conf. on Knowledge Discovery and Data Mining (PAKDD), 2002.

[14]H. Grundel, T. Naphtali, C. Wiech, J.-M. Gluba, M. Rohdenburg, and T. Scheffer. Clipping and analyzing news using machine learning techniques. Proc. of the International Conf. on Discovery Science. 2001.

[15]C. N. Hsu and M. T. Dung. Generating Finite-state Transducers for Semi-structured Data Extraction from the Web. Information Systems, 23(8):521-538, 1998.

[16]H.-Y. Kao, S. H. Lin, J. M. Ho and M.-S. Chen. Entropy-Based Link Analysis for Mining Web Informative Structures. Proc. of the ACM 11th International Conf. on Information and Knowledge Management (CIKM), 2002.

[17]H.-Y. Kao, S.-H. Lin, J.-M. Ho and M.-S. Chen, Mining Web Information Structures and Contents based on Entropy Analysis, IEEE Trans. on Knowledge and Data Engineering, Vol. 16, No. 1, January 2004.

[18]J. M. Kleinberg, Authoritative sources in a hyperlinked environment. ACM-SIAM Symposium on Discrete Algorithms (SODA), 1998.

[19] N. Kushmerick, D. Weld, and R. Doorenbos, Wrapper Induction for Information Extraction, Proc. of the $15^{\text {th }}$ International Joint Conf. on Artificial Intelligence (IJCAI), 1997. 
[20]A. Laender, B. Ribeiro-Neto, A. Silva, and J. Teixeira, A Brief Survey of Web Data Extraction Tools, SIGMOD Record Vol. 31, Number 2, June 2002.

[21]S. H. Lin and J. M. Ho. Discovering Informative Content Blocks from Web Documents. The 8th ACM International Conf. on Knowledge Discovery and Data Mining (SIGKDD), 2002.

[22] W. Y. Lin, W. Lam. Learning to Extract Hierarchical Information from Semi-structured Documents. Proc. of the ACM 9th International Conf. on Information and Knowledge Management (CIKM), 2000.

[23]X. Li, B. Liu, T.-H. Phang, M. Hu, "Using Micro Information Units for Internet Search", Proc. of the ACM 11th International Conf. on Information and Knowledge Management (CIKM), 2002.

[24] T. Miyahara, Y. Suzuki, T. Shoudai, T. Uchida, K. Takahashi, H. Ueda. Discovery of Frequent Tag Tree Patterns in Semistructured Web Documents. Proc. of the Sixth Pacific-Asia Conf. on Knowledge Discovery and Data Mining (PAKDD), 2002.

[25]C. E. Shannon, A mathematical theory of communication. Bell System Technical Journal, 27:398403, 1948.

[26] G. Salton, Automatic Text Processing: The Transformation, Analysis, and Retrieval of Information by Computer. Addison Wesley. 1989.

[27] W3C DOM. Document Object Model (DOM). http://www.w3.org/DOM/.

[28]K. Wang, H. Liu. Discovering Structural Association of Semistructured Data. IEEE Transaction on Knowledge and Engineering, Vol. 12, No. 3, MAY/JUNE 2000.

[29]C. Yip, C. Gertz, and N. Sundaresan. Reverse Engineering for Web Data: From Visual to Semantic Structures. Proc. of the 19th IEEE International Conf. on Data Engineering (ICDE), 2002. 


\section{APPENDIX A}

[Proof 1]: Prove relationships between $\mathrm{W}\left(T_{j}\right)$ and $I D F_{j}$ : (1) If $T_{j}$ is uniformly distributed among some pages, $\mathrm{W}\left(T_{j}\right)=I D F_{j}$, and (2) If $T_{j}$ is not uniformly distributed among the same pages in (1), then $\mathrm{W}\left(T_{j}\right)>$ $I D F_{j}$ and the more skewed the distribution of $T_{j}$ is, the larger $\mathrm{W}\left(T_{j}\right)$ is.

Relationship (1): If $T_{j}$ is uniformly distributed among some of pages, $\mathrm{W}\left(T_{j}\right)=I D F_{j}$

Proof: assume $\mathrm{n}=|\mathrm{D}|, t f_{j}=$ the total frequency of $T_{j}$, because $T_{j}$ is uniformly distributed, $t f_{i j}$, i.e., the term frequency of $T_{j}$ in page $\mathrm{i}_{\mathrm{i}}$, is equal to $\frac{t f_{j}}{d f_{j}}$. According to the definition of the entropy-based weighting, $W\left(T_{j}\right)=1-E N\left(T_{j}\right)=1-\left(-\sum_{\mathrm{df}_{\mathrm{j}}} \frac{t f_{i j}}{t f_{j}} \log _{n} \frac{t f_{i j}}{t f_{j}}\right) \quad$ and $\quad t f_{j}=t f_{i j} * d f_{j} . \quad$ Therefore, we can conclude $W\left(T_{j}\right)=1+\log _{n} \frac{1}{d f_{j}}=\log _{n} \frac{n}{d f_{j}}=I D F_{j}$.

Relationship (2): If $T_{j}$ is not uniformly distributed, then $\mathrm{W}\left(T_{j}\right)>I D F_{j}$

Proof: When the distribution of $T_{j}$ is more skewed, $E N\left(T_{j}\right)$ will decrease according to Information Theory. $\mathrm{W}\left(T_{j}\right)$ will therefore increase, but $d f_{j}$ is constant under the assumption. The relationship (2) is therefore conformed.

[Proof 2]: Prove $E_{k+1}\left(f_{j}\right)=\left\{\begin{array}{l}\frac{E_{k}\left(f_{j}\right)}{\log _{k}(k+1)}, \text { when } t f_{(k+1) j}=0 \\ \Phi\left(E_{k}\left(f_{j}\right), \quad T F_{j, k}, \quad t f_{(k+1) j}\right), \text { otherwise }\end{array}\right.$

Proof: Suppose we have obtained the entropy value $E_{k}\left(f_{j}\right)$ for term $f_{j}$ from the page set containing $k$ Web pages by Equation A.1.

$$
E_{k}\left(f_{j}\right)=-\sum_{i=1}^{k} \frac{t f_{i j}}{T F_{j, k}} \log _{k} \frac{t f_{i j}}{T F_{j, k}} \cdots(A .1)
$$


where $t f_{i j}$ means the term frequency of $f_{j}$ in page $P_{i}$ and $T F_{j, k}$ is equal to $\sum_{i=1}^{k} t f_{i j}$. When a new page is added into the page set, the new entropy value $E_{k+1}\left(f_{j}\right)$ can be calculated by the following equation.

$$
\begin{aligned}
& E_{k+1}\left(f_{j}\right)=-\sum_{i=1}^{k+1} \frac{t f_{i j}}{T F_{j, k+1}} \log _{k+1} \frac{t f_{i j}}{T F_{j, k+1}}=\frac{1}{T F_{j, k+1}}\left(-\sum_{i=1}^{k+1} t f_{i j} \log _{k+1} t f_{i j}+\sum_{i=1}^{k+1} t f_{i j} \log _{k+1} T F_{j, k+1}\right) \\
& =\frac{1}{T F_{j, k+1}}\left(-\sum_{i=1}^{k+1} t f_{i j} \frac{\log _{k} t f_{i j}}{\log _{k}(k+1)}+\sum_{i=1}^{k+1} t f_{i j} \log _{k+1} T F_{j, k+1}\right) \\
& =\frac{1}{T F_{j, k+1}}\left(-\frac{1}{\log _{k}(k+1)}\left(\sum_{i=1}^{k} t f_{i j} \log _{k} t f_{i j}+t f_{(k+1) j} \log _{k} t f_{(k+1) j}\right)+\sum_{i=1}^{k+1} t f_{i j} \log _{k+1} T F_{j, k+1}\right) \\
& =\frac{1}{T F_{j, k+1}}\left(-\frac{1}{\log _{k}(k+1)}\left(\sum_{i=1}^{k} t f_{i j} \log _{k} T F_{j, k}-T F_{j, k} * E_{k}\left(f_{j}\right)+t f_{(k+1) j} \log _{k} t f_{(k+1) j}\right)+\sum_{i=1}^{k+1} t f_{i j} \log _{k+1} T F_{j, k+1}\right) \\
& =\frac{1}{T F_{j, k+1}}\left(\frac{1}{\log _{k}(k+1)}\left(T F_{j, k} * E_{k}\left(f_{j}\right)-t f_{(k+1) j} \log _{k} t f_{(k+1) j}\right)-\sum_{i=1}^{k} t f_{i j} \log _{k+1} T F_{j, k}+\sum_{i=1}^{k+1} t f_{i j} \log _{k+1} T F_{j, k+1}\right) \\
& =\frac{1}{T F_{j, k+1}}\left(\frac{T F_{j, k} * E_{k}\left(f_{j}\right)}{\log _{k}(k+1)}-t f_{(k+1) j} \log _{k+1} t f_{(k+1) j}-T F_{j, k} \log _{k+1} T F_{j, k}+T F_{j, k+1} \log _{k+1} T F_{j, k+1}\right) \\
& =\frac{1}{T F_{j, k+1}}\left(\frac{T F_{j, k} * E_{k}\left(f_{j}\right)}{\log _{k}(k+1)}-t f_{(k+1) j} \log _{k+1} t f_{(k+1) j}+t f_{(k+1) j} \log _{k+1} T F_{j, k+1}+T F_{j, k}\left(\log _{k+1} T F_{j, k+1}-\log _{k+1} T F_{j, k}\right)\right. \\
& =\frac{1}{T F_{j, k}+t f_{(k+1) j}}\left(\frac{T F_{j, k} * E_{k}\left(f_{j}\right)}{\log _{k}(k+1)}+t f_{(k+1) j} \log _{k+1}\left(1+\frac{T F_{j, k}}{t f_{(k+1) j}}\right)+T F_{j, k} \log _{k+1}\left(1+\frac{t f_{(k+1) j}}{T F_{j, k}}\right)\right) \ldots(A .2)
\end{aligned}
$$

Therefore, we can conclude that $E_{k+1}\left(f_{j}\right)=\left\{\begin{array}{l}\frac{E_{k}\left(f_{j}\right)}{\log _{k}(k+1)}, \text { when } t f_{(k+1) j}=0 \\ \Phi\left(E_{k}\left(f_{j}\right), \quad T F_{j, k}, \quad t f_{(k+1) j}\right), \text { otherwise }\end{array}\right.$. 